\title{
ON COMPLETE CONVERGENCE OF THE SUM OF A RANDOM NUMBER OF STABLE TYPE P RANDOM ELEMENTS
}

\author{
ANDRÉ ADLER \\ Department of Mathematics \\ Illinois Institute of Technology \\ Chicago, IL 60616 U.S.A. \\ and \\ ANDREY VOLODIN \\ Tresearch Institute of Mathematics \\ Kazan University \\ Kazan Tatarstan \\ Russia 420008 \\ (Received September 1, 1992 and in revised form March 10, 1993)
}

ABSTRACT. Complete convergence for randomly indexed normalized sums of random elements of the form $\sum_{\jmath=1}^{T_{n}} X_{\jmath} / \phi\left(T_{n}\right)$ is established. The random elements $\left\{X_{n}\right\}$ belong to a type p stable space and are assumed to be independent, but not necessarily identically distributed. No assumptions are placed on the joint distributions of the stopping times $\left\{T_{n}\right\}$

KEY WORDS AND PHRASES. Complete convergence, stable type p. 1990 AMS SUBJECT CLASSIFICATION CODES. 60F15, 60B12.

In this article we extend previous results on complete convergence for randomly stopped sums. A sequence of random elements $\left\{X_{n}\right\}$ is said to converge completely to zero if for all $\epsilon>0$

$$
\sum_{n=1}^{\infty} P\left\{\left\|X_{n}\right\|>\epsilon\right\}<\infty .
$$

In view of the Borel-Cantelli lemma it's clear that complete convergence implies almost sure convergence. Hsu and Robbins [5] are credited with the introduction of this concept. Since then many well known mathematicians have explored this interesting subject.

This paper generalizes the work of Adler [1] and Gut [3] via methods that can be found in Volodin [7]. We extend both articles in a couple of ways. First of all our random variables are no longer solely defined on the real line. Also, we introduce the function $\phi(x)$, which we use to normalize the partial sums. Hence, the norming sequence is not just the number of terms in our partial sum, but a function of the number of terms. One should compare Theorem 1 with Theorem 4.1 of Gut [3] and Theorem 2 with Theorem 2 of Adler $[1]$

The first definition that we need to introduce is that of a stable type $p$ Banach space. Let $\left\{\gamma_{n}\right\}$ be a sequence of independent random variables with characteristic function $\exp \left\{-|t|^{p}\right\}$, where $1 \leq p<2$. The Banach space $\mathrm{E}$ is said to be of stable type $\mathrm{p}$ if 
for all $X_{n} \subset E$ the convergence of $\sum_{n=1}^{x}\left\|X_{n}\right\|^{p}$ implies the alnost sure convergence of $\sum_{n=1}^{\infty} \gamma_{n} \mathrm{X}_{n}$. Set $p(E)=\sup \{p: E$ is of stable type $p\}$. Note that since the interval of stable types is open and $1 \leq p<2$ we can select a number $\mathrm{r}$ such that $p<r<p(E)$.

Instead of the numal hypothesis. that our random elenents all have the same distribution. we assume that there is a dominating random variable, i.e. we say that $X$ dominates $\left\{X_{n}\right\}$ if there exists a constant $D$ so that

$$
\sup _{n \geq 1} P\left\{\left\|X_{n}\right\|>t\right\} \leq D P\{X>t\} \quad \text { for all } t>0 .
$$

Next, let $\left\{a_{n}\right\}$ be a sequence of strictly increasing integers. Also, let $\{\phi(n)\}$ be a sequence of positive constants such that $\phi\left(\alpha_{n}\right) \geq a \alpha_{n}^{1 / p}$ for all $n$ and some positive constant a. As usual set $M(t)=\sum_{n=1}^{[t]} \alpha_{n}, \Phi(t)=\operatorname{card}\left\{n: \alpha_{n} \leq t\right\}$ and $\psi(t)=\operatorname{card}\{n: \phi(n) \leq t\}$. Set $S_{n}=\sum_{\jmath=1}^{n} X_{J}$. Finally, note that the constant $C$ is a generic constant which is not necessarily the same in each appearance. In most situations we are only concerned with obtaining upper bounds to our terms. Hence when one term is majorized by another we combine all the coefficients into one, which we denote by $C$.

Our first result exhibits a classical strong law. It is classical in the sense that the stopping times are not random. It will be of great value in proving Theorem 2 .

THEOREM 1. Let $\mathrm{E}$ be a Banach space of stable type $\mathrm{p}$, where $1 \leq p<2$. Let $\left\{X_{n}\right\}$ be a sequence of independence mean zero random elements in $\mathrm{E}$. Let $\mathrm{X}$ be a positive random variable that dominates $\left\{X_{n}\right\}$. If $E X^{r}<\infty$, where $p<r<p(E)$ and $E M(\Phi(\Psi(2 X)))<$ $\infty$, then $S_{\alpha_{n}} / \phi\left(\alpha_{n}\right)$ converges completely to zero.

PROOF. Without loss of generality we may assume that $\left\{X_{n}\right\}$ is a sequence of symmetric random elements. Since $r>p$ we can select a number $m$ so that $(r / p-1) 2^{m}$ exceeds one. By reapplying Hoffmann-Jorgensen's [4] and Chebyshev's inequalities we have for all $\epsilon>0$

$$
\begin{aligned}
\sum_{n=1}^{\infty} P\left\{\left\|S_{\alpha_{n}}\right\|>\epsilon 3^{m} \phi\left(\alpha_{n}\right)\right\} & \leq C \sum_{n=1}^{\infty}\left[P\left\{\left\|S_{\alpha_{n}}\right\|>\epsilon \phi\left(\alpha_{n}\right)\right\}\right]^{2^{m}} \\
& +C \sum_{n=1}^{\infty} \max _{1 \leq j \leq \alpha_{n}} P\left\{\|X,\|>\epsilon \phi\left(\alpha_{n}\right)\right\} \\
& \leq C \sum_{n=1}^{\infty}\left[\epsilon^{-r} \phi^{-r}\left(\alpha_{n}\right) E\left\|S_{\alpha_{n}}\right\|^{r}\right]^{2^{m}} \\
& +C \sum_{n=1}^{\infty} \alpha_{n} P\left\{X>\epsilon \phi\left(\alpha_{n}\right)\right\} \\
& \leq C \sum_{n=1}^{\infty}\left[\phi^{-r}\left(\alpha_{n}\right) \sum_{j=1}^{\alpha_{n}} E\|X,\|^{r}\right]^{2^{m}} \\
& +C E M(\Phi(\Psi(X))) \\
& \leq C\left(E X^{r}\right)^{2^{m}} \sum_{n=1}^{\infty}\left[\alpha_{n} \phi^{-r}\left(\alpha_{n}\right)\right]^{2^{m}}+O(1)
\end{aligned}
$$




$$
\begin{aligned}
& \leq C \sum_{n=1}^{\infty} n^{-(r / p-1) 2^{m}}+O(1) \\
& =O(1) .
\end{aligned}
$$

which completes the proof.

Next we introduce our stopping times. Let $\left\{T_{n}\right\}$ be a sequence of integer valued random variables. The ensuing theorem establishes a strong law for a randomly stopped sum.

THEOREM 2. In addition to the hypotheses of Theorem 1 suppose that $\left\{\beta_{n}\right\}$ is a sequence of positive constants with $\lim \sup _{n \rightarrow \infty} \beta_{n}<1, \sum_{n=1}^{\infty} \beta_{n} \phi^{p-r}\left(\alpha_{n}\right)<\infty$ and $\sum_{n=1}^{\infty} P\left\{\left|T_{n} / \alpha_{n}-1\right|>\beta_{n}\right\}<\infty$. If $\phi\left(2 \alpha_{n}\right)<b \phi\left(\alpha_{n}\right)$ for all $n$ and some constant $b$, then $S_{T_{n}} / \phi\left(T_{n}\right)$ converges completely to zero.

PROOF. In view of Theorem 1 we may conclude that $S_{\alpha_{n}} / \phi\left(\alpha_{n}\right)$ converges completely to zero. Next, we show that $\left(S_{T_{n}}-S_{\alpha_{n}}\right) / \phi\left(\alpha_{n}\right)$ converges to zero, which implies that $S_{T_{n}} / \phi\left(\alpha_{n}\right)$ converges completely to zero.

Set $A_{n}=\left\{k:\left[\alpha_{n}\left(1-\beta_{n}\right)\right]+1 \leq k \leq \alpha_{n}-1\right\}$ and $B_{n}=\left\{k: \alpha_{n}+1 \leq k \leq\left[\alpha_{n}\left(1+\beta_{n}\right)\right]\right\}$. Let $\epsilon>0$. By applying Etemadi's [2] maximal inequality and Cheyshev's inequality we have

$$
\begin{aligned}
& \sum_{n=1}^{\infty} P\left\{\left\|S_{T_{n}}-S_{\alpha_{n}}\right\|>\epsilon \phi\left(\alpha_{n}\right)\right\} \\
& \leq \sum_{n=1}^{\infty} P\left\{\left\|S_{T_{n}}-S_{\alpha_{n}}\right\|>\epsilon \phi\left(\alpha_{n}\right),\left|\frac{T_{n}}{\alpha_{n}}-1\right| \leq \beta_{n}\right\} \\
& +\sum_{n=1}^{\infty} P\left\{\left|\frac{T_{n}}{\alpha_{n}}-1\right|>\beta_{n}\right\} \\
& \leq \sum_{n=1}^{\infty} P\left\{\max _{\alpha_{n}\left(1-\beta_{n}\right) \leq k \leq \alpha_{n}\left(1+\beta_{n}\right)}\left\|S_{k}-S_{\alpha_{n}}\right\|>\epsilon \phi\left(\alpha_{n}\right)\right\}+O(1) \\
& \leq \sum_{n=1}^{\infty} P\left\{\max _{k \in A_{n}}\left\|\sum_{j=k+1}^{\alpha_{n}} X,\right\|>\epsilon \phi\left(\alpha_{n}\right)\right\} \\
& +\sum_{n=1}^{\infty} P\left\{\max _{k \in B_{n}}\left\|\sum_{j=\alpha_{n}+1}^{k} X,\right\|>\epsilon \phi\left(\alpha_{n}\right)\right\}+O(1) \\
& \leq C \sum_{n=1}^{\infty} \max _{k \in A_{n}} P\left\{\left\|\sum_{j=k+1}^{\alpha_{n}} X,\right\|>\epsilon \phi\left(\alpha_{n}\right) / 4\right\} \\
& +C \sum_{n=1}^{\infty} \max _{k \in B_{n}} P\left\{\left\|\sum_{j=\alpha_{n}+1}^{k} X_{j}\right\|>\epsilon \phi\left(\alpha_{n}\right) / 4\right\}+O(1) \\
& \leq C \sum_{n=1}^{\infty} \phi^{-r}\left(\alpha_{n}\right)\left[\max _{k \in A_{n}} E\left\|\sum_{j=k+1}^{\alpha_{n}} X,\right\|^{r}+\max _{k \in B_{n}} E\left\|\sum_{j=\alpha_{n}+1}^{k} X,\right\|^{r}\right]+O(1) \\
& \leq C \sum_{n=1}^{\infty} \phi^{-r}\left(\alpha_{n}\right) \sum_{\jmath=\left[\alpha_{n}\left(1-\beta_{n}\right)\right]+2}^{\left[\alpha_{n}\left(1+\beta_{n}\right)\right]} E\|X,\|^{r}+O(1) \\
& \leq C E X^{r} \sum_{n=1}^{\infty} \alpha_{n} \beta_{n} \phi^{-r}\left(\alpha_{n}\right)+O(1)
\end{aligned}
$$




$$
\begin{aligned}
& \leq C \sum_{n=1}^{\infty} \beta_{n} \phi^{p-r}\left(\alpha_{n}\right)+O(1) \\
& =O(1) .
\end{aligned}
$$

From the hypothesis $\phi\left(2 \alpha_{n}\right) \leq b \phi\left(\alpha_{n}\right)$ it follows that for all $0<\delta<1: \phi\left(\delta \alpha_{n}\right) \geq$ $C \phi\left(\alpha_{n}\right)$. Next, choose $\beta$ and $N$ so that $\beta_{n}<\beta<1$ for all $n \geq N$. Observe that

$$
\begin{aligned}
\sum_{n=1}^{\infty} P\left\{\left\|S_{T_{n}}\right\|>\epsilon \phi\left(T_{n}\right)\right\} & =\sum_{n=N}^{\infty} P\left\{\left\|S_{T_{n}}\right\|>\epsilon \phi\left(T_{n}\right)\right\}+O(1) \\
& \leq \sum_{n=N}^{\infty} P\left\{\left\|S_{T_{n}}\right\|>\epsilon \phi\left(T_{n}\right),\left|\frac{T_{n}}{\alpha_{n}}-1\right| \leq \beta_{n}\right\} \\
& +\sum_{n=N}^{\infty} P\left\{\left|\frac{T_{n}}{\alpha_{n}}-1\right|>\beta_{n}\right\}+O(1) \\
& \leq \sum_{n=N}^{\infty} P\left\{\left\|S_{T_{n}}\right\|>\epsilon \phi\left(T_{n}\right), T_{n}>\alpha_{n}(1-\beta)\right\}+O(1) \\
& \leq \sum_{n=N}^{\infty} P\left\{\left\|S_{T_{n}}\right\|>\epsilon \phi\left(\alpha_{n}(1-\beta)\right)\right\}+O(1) \\
& \leq \sum_{n=N}^{\infty} P\left\{\left\|S_{T_{n}}\right\|>C \epsilon \phi\left(\alpha_{n}\right)\right\}+O(1) \\
& =O(1)
\end{aligned}
$$

which completes the proof.

For more on recent results on complete convergence for Banach valued random elements that also explores the issue of randomly indexed sums see Kuczmaszewska and Szynal [6].

\section{REFERENCES}

1. ADLER, A. On complete convergence of the sum of a random number of random variables, Calcutta Statist. Assoc. Bull. 37 (1988), 161-169.

2. ETEMADI, N. On some classical results in probability theory, Sankhya ser. A 47 (1983), 215-221.

3. GUT, A. On complete convergence in the law of large numbers for subsequences, Ann Probab. 13 (1985), 1286-1291.

4. HOFFMANN-JORGENSEN, J. Sums of independent Banach space valued random variables, Studia Math. 11 (1974), 159-186.

5. HSU, P.L. and ROBBINS, H. Complete convergence and the law of large numbers, Proc. Nat. Acad. Sci. U.S.A. 33 (1947), 25-31.

6. KUCZMASZEWSKA, A. and SZYNAL, D. On complete convergence in a Banach space, Inter. J. Math. and Math. Sci. 16 (1993).

7. VOLODIN, A. On $B_{\phi}$-convex spaces, Izvestia VUZ Mathematica 4 (1992), 3-12. 


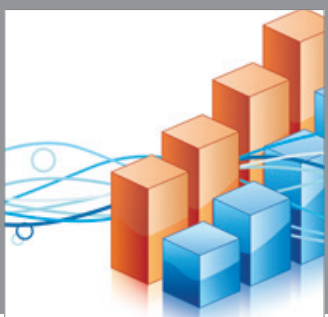

Advances in

Operations Research

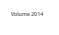

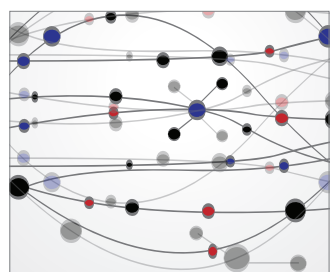

\section{The Scientific} World Journal
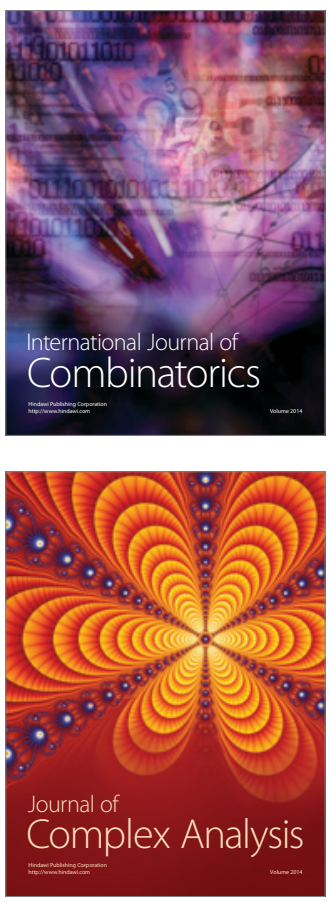

International Journal of

Mathematics and

Mathematical

Sciences
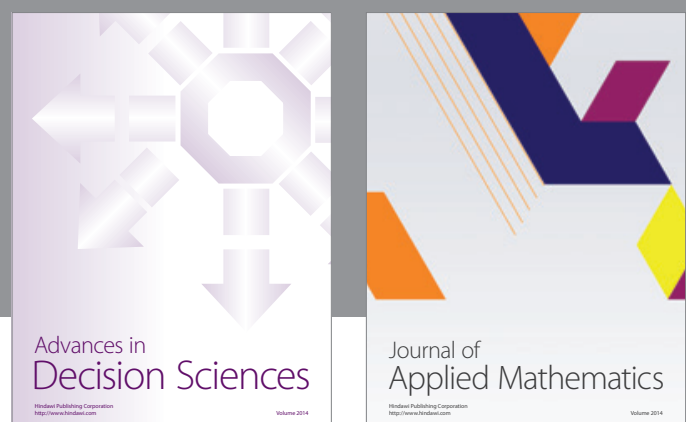

Journal of

Applied Mathematics
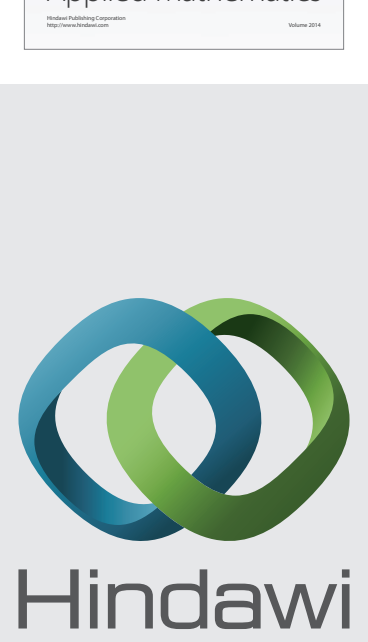

Submit your manuscripts at http://www.hindawi.com
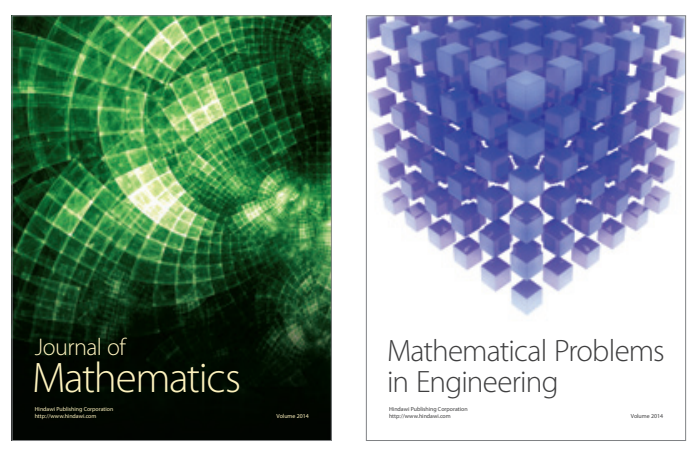

Mathematical Problems in Engineering
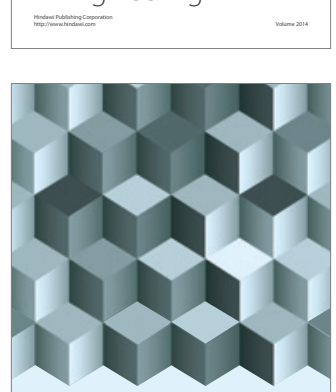

Journal of

Function Spaces
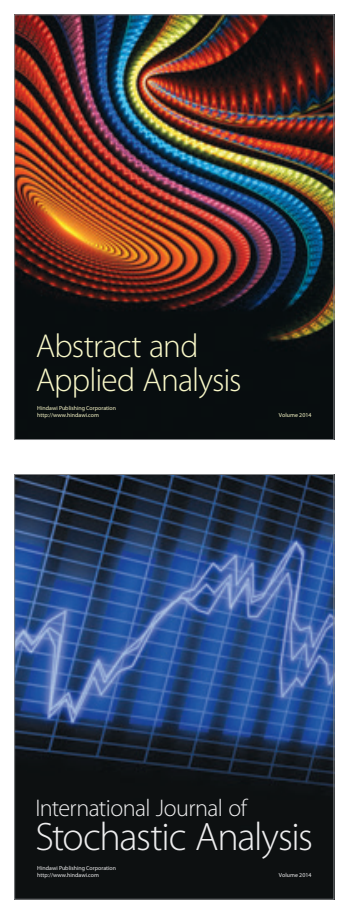

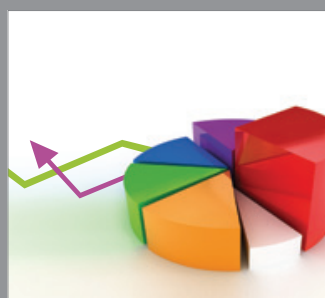

ournal of

Probability and Statistics

Promensencen
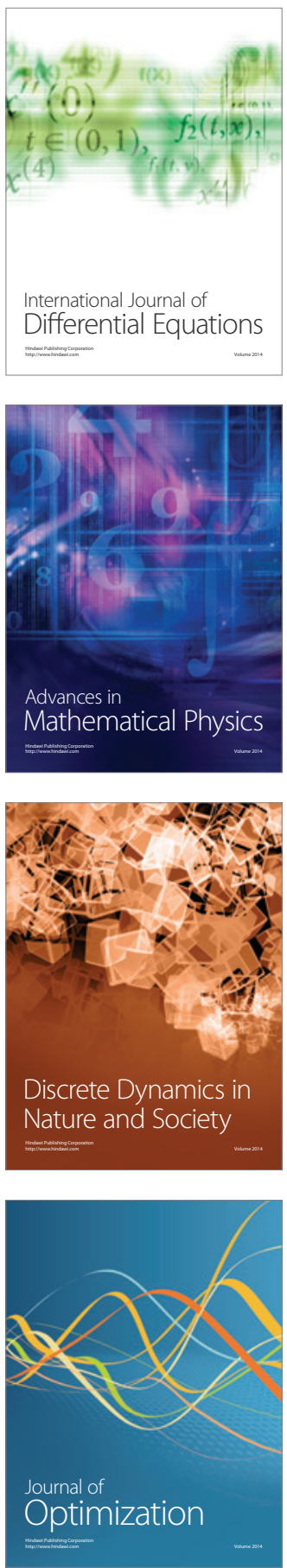